\title{
Suggestion for New Classes of IP Addresses
}

Status of this Memo

This memo provides information for the Internet community. It does not specify an Internet standard. Distribution of this memo is unlimited.

Abstract

This RFC suggests a change in the method of specifying the IP address to add new classes of networks to be called F, G, H, and $K$, to reduce the amount of wasted address space, and to increase the available IP address number space, especially for smaller organizations or classes of connectors that do not need or do not want a full Class C IP address.

Table of Contents

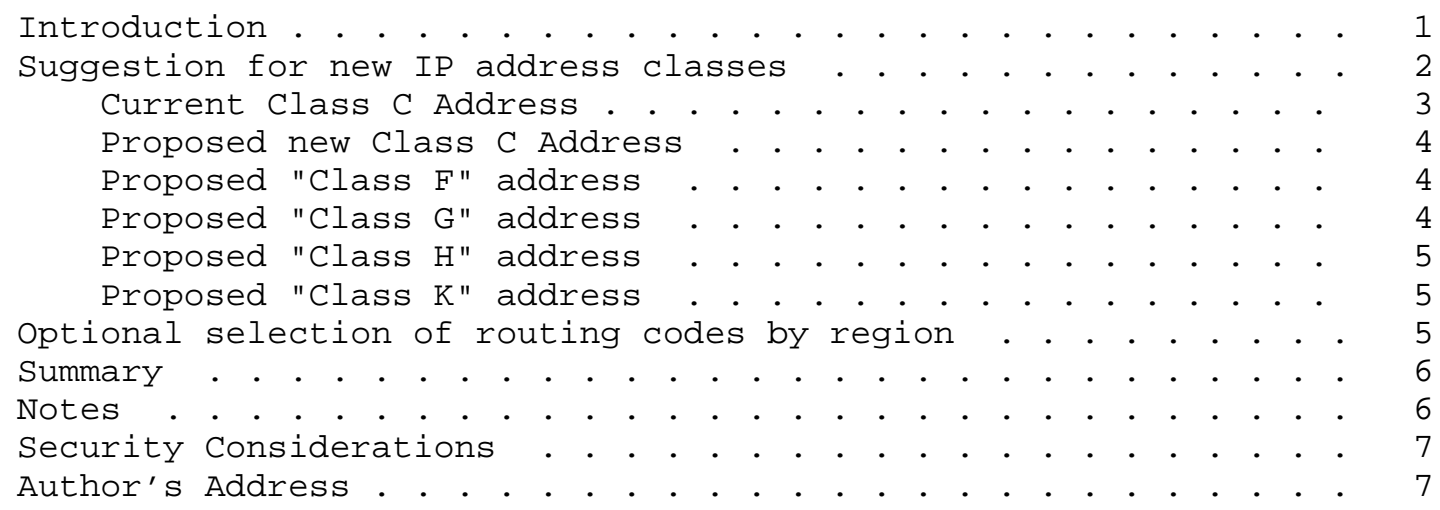

Introduction

Currently, IP addresses on the Internet are 32-bit quantities which are generally represented as four decimal numbers from 0 to 255, separated by periods, sometimes called a "dotted" decimal number. The current numbering scheme provides in general for three classes of networks in general use (A, B, and C), and two other classes of networks (D, E).

The Class A networks assign a large address space for the particular 
network to allow up to 254^3 local machines [1]. The Class B network assigns a somewhat smaller address space for the particular network to allow up to 254^2 local machines. The Class C network assigns a still smaller address space for the particular network to allow up to 254 local machines.

This memo proposes to assign part of the unused Class C address space for smaller networks than are currently available. The term "Class D" is used for the "multicast" capability and addresses in "Class E" are reserved for future use. Therefore, these new features for which capability is to be added is being referred to as classes F, G, H and $\mathrm{K}$.

Suggestion for new IP address classes

The most worrisome problem which appears in the literature is the possibility of running out of address space for IP addresses. Various schemes are being suggested such as subrouting, introduction of additional bits, and other possibilities.

There is an even more serious matter. In all probability, I suspect that eventually the Internet backbone will either become available to anyone who wants to use it (like public highways) and the costs paid for out of taxes or some other method which gets someone else to pay for it, or eventually the Internet will be fully commercialized and made available to anyone who wants to buy a permanent connection. With the cost of hardware and connections dropping, some Computer Bulletin Board Systems (BBSs) which are currently accessible via telephone call may become accessible via TELNET or FTP. When a 9600 baud connection can be obtained for around the price of a phone line, the demand for internet access will skyrocket. This almost certain eventual availability to virtually anyone who wants a connection will cause an even greater demand for internet addresses, which will exacerbate this situation. One problem is in the granularity of IP addressing, in that the smallest possible IP address one may obtain allows for as high as 254 IP addresses. If someone wanted only to put four or five computers on the Internet, more than 240 addresses are wasted.

Many smaller installations would probably be interested either in placing their computers and/or servers on the Internet (and perhaps helping to pay the cost of running it) or in being able to access the Internet directly, and perhaps making facilities on their machines available to others; the problem being that IP addresses on Internet are not readily available to small classes of users. Also, the possibility exists of eventually placing non-computer and output-only devices such as printers, facsimile machines, and visual pagers directly on the Internet to allow people to send a message to a local 
device simply by directing it to a specific internet site as an EMail message.

The scheme proposed by this paper proposes to make a slight change in one of the classes of network address in a manner which should not be a significant problem for implementing, and should not cause a significant hardship as the addresses to use for this purpose are not now allocated anyway, and may draw some of the drain which would have consumed Class $\mathrm{C}$ addresses in large quantity into quantities of Class $\mathrm{F}, \mathrm{H}$, or $\mathrm{K}$ addresses which waste less IP address space.

This scheme I am proposing is to allow for very small networks (1 or 2, 1-7, or 1-15, depending on the number of addresses the administrator of that site thinks he will need), by reconstructing the network address to include what is nominally part of the local address. If bridges and routers (and other hardware and software) do not assume that only the last 8 bits make up a local address and permit smaller spaces for local addresses, then this method should not cause problems. Sites needing less than a close order of 256 IP addresses could simply apply for 2 or more contiguous blocks of Class F numbers.

Currently, a class C address consists of a 32-bit number in which the leftmost 3 bits consist of "110" [2]:

The third type of address, class C, has a 21-bit network number and a 8-bit local address. The three highest-order bits are set to 1-1-0. This allows 2,097,152 class C networks.

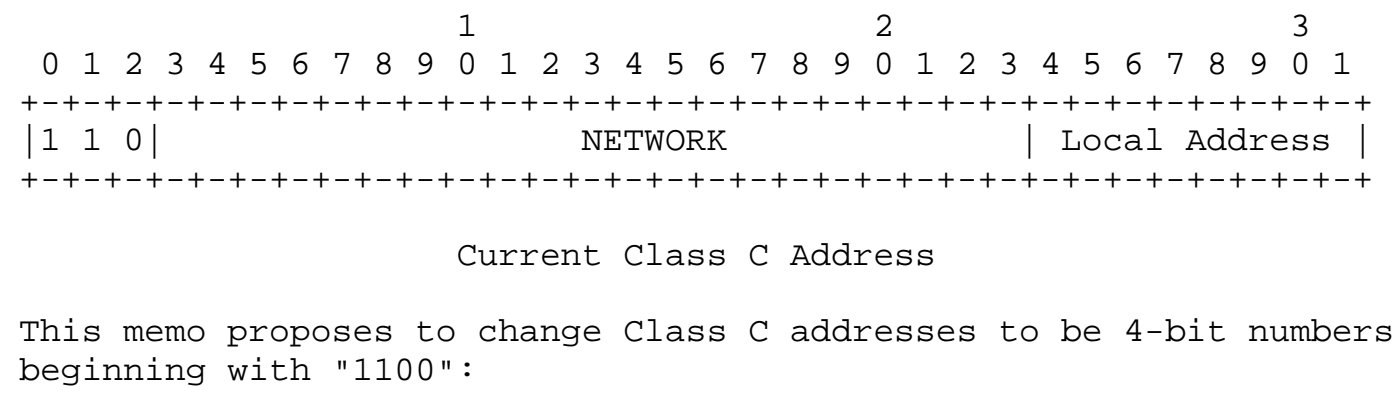

The third type of address, class C, has a 20-bit network number and a 8-bit local address. The four highest-order bits are set to 1-1-0-0, This allows 1,048,576 class C networks. 


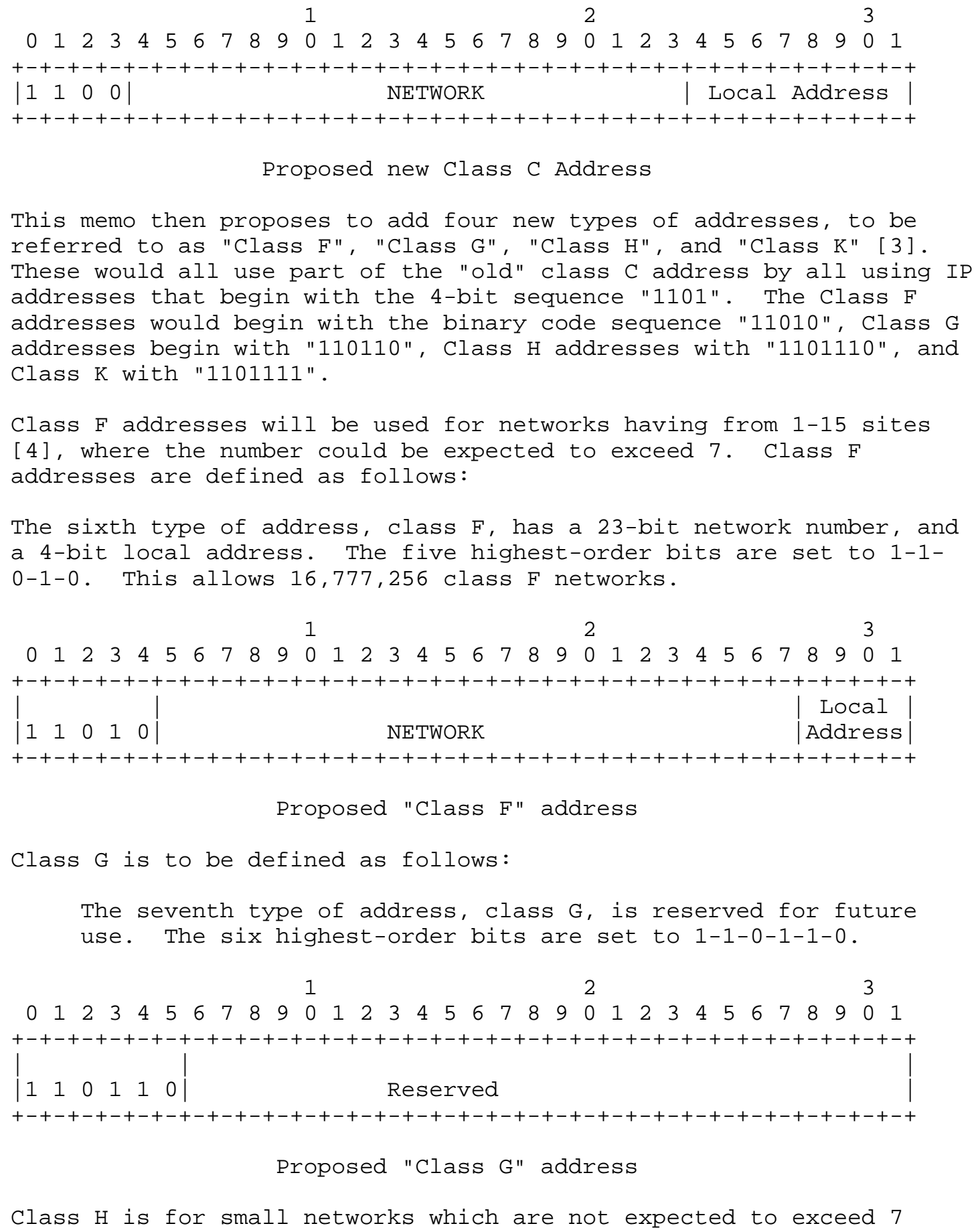


connected IP addresses. Class $\mathrm{H}$ is to be defined as follows:

The eighth type of address, class H, has a 22-bit network number, and a 3-bit local address. The seven highest-order bits are set to 1-1-0-1-1-1-0. This allows 4,194,304 Class H addresses [5].

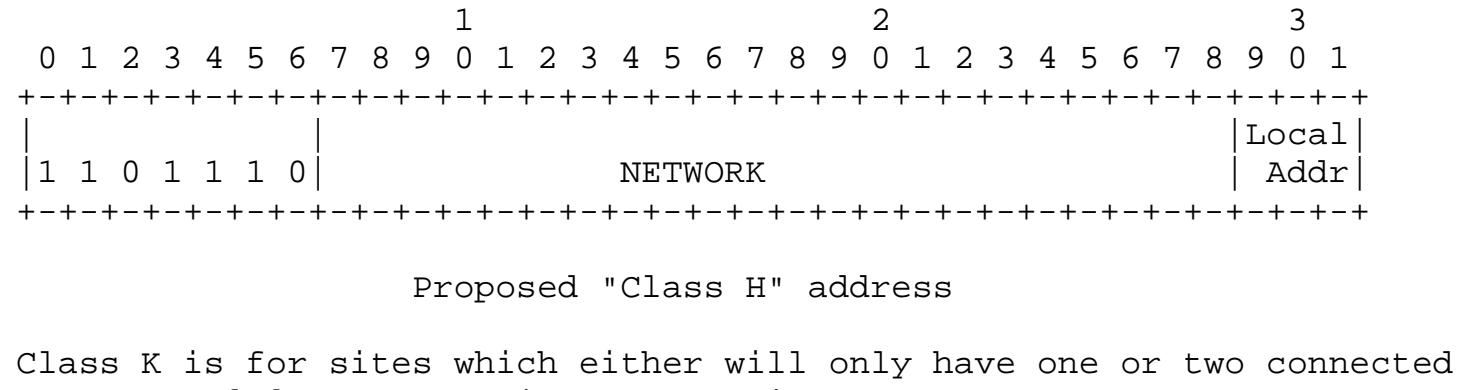

Optional selection of routing codes by region

Because of the possibility of confusion, some method similar to the international dialing plan might be set up, in which bits 5-8 in Class F, bits 7-10 in Class H, and bits 6-9 in Class $\mathrm{K}$ could be used to define what part of the world the particular address is in, in a manner similar to the international telephone dialing system, which uses the first digit of the international telephone number to determine the region being used. The current method for assigning international dialing codes is: 
1 North America

2 Africa

3 Europe

4 Europe

5 South America and Mexico
6 Oceania, Australia

7 Ex-Soviet Union Countries

8 Asia

9 Mideast

If a similar method is used, I would recommend assigning 0,1,10 and 11 to North America, 8 and 12 to Asia, and leaving 13 through 15 for other areas as needed. Note that this would simply make some routing choices easier, it is not precisely necessary that this be done, since currently routing is generally done using the shortest path to a site and IP numbers don't really relate to any specific address anywhere in the world.

The number form of a class F, G, H or K address could still be listed in the standard form n.n.n.n, as long as it is not assumed that the 4th chunk number alone identifies a local address and that numbers with the same preceding 3 chunks do not necessarily belong to the same network.

Summary

In order to make the address space available, even if the method to implement this feature is not presently available, it is suggested that Class F, G, H, and K address space should be taken out of Class $C$ space and reserved for the purpose of allowing smaller-sized networks so that this feature may be made available. Since Class C addresses currently are only using the equivalent of one Class A number anyway, this should not cause a problem.

Notes

[1] Common practice dictates that neither an address 0 nor 255 should be used in any "dotted" address.

[2] Reynolds, J., and J. Postel, "Assigned Numbers", STD 2, RFC 1340, USC/Information Sciences Institute, July 1992.

[3] To prevent confusion, no class "I" or "J" address was created by this memo.

[4] It is expected that if the higher part of the network address occupying the 4-bits to the left of the Class $F$ address are neither 0 nor 15, that a class $F$ site could have 16 machines. If the 4-bits to the left are all 0, the Class F site must not use number 0 . If the four bits are all 1 , the site must not use number 15 . 
[5] It may be that Class $H$ numbers are more appropriate for classes of addresses that will not have as high a demand for access via Internet addresses such as facsimile machines and pagers. (The end digit could be used to determine class of service, i.e., 0 for tone only, 1 and 2 for numeric only, 34 and 5 for alphanumeric, and 6 and 7 for facsimile machines. Or some combination of these according to the demand. Remember, Internet won't always be just text messages and file transfers; we may eventually see things like voice telephone calls or voice data being placed to an Internet address just like calls made via the telephone system. This would require a whole change in the way things are done, but it's always best to look at the future.

[6] It is suggested that addresses in this range not be assigned where the 7 bits to the left of the local number are all the same (all 0 or all 1), to allow all Class $\mathrm{K}$ addresses to have two local addresses.

[7] Different things can be done with different capabilities. One thought was to set up some group of numbers and use them to indicate systems which are "gateway" systems, i.e., the top set of numbers in Class $\mathrm{K}$ could indicate that subnets are required after those numbers, similar to the use of an extension number on the switchboard of a large organization. Another possibility is to assign some of the numbers to specific classes of devices, such as number-only pagers and electronic display devices.

Security Considerations

Security issues are not discussed in this memo.

Author's Address

Paul Robinson

Tansin A. Darcos \& Company

8604 Second Avenue \#104

Silver Spring, MD 20910 USA

Phone: 202-310-1011

Telex: 6505066432MCI UW

E-mail: TDARCOS@MCIMAIL.COM 